\title{
Serum folates in man
}

\author{
K. RAtANAS THIEN, J. A. BLAiR, R. J. LEEMING, W. T. COOKE, AND \\ V. MELIKIAN
}

From the Department of Chemistry, University of Aston in Birmingham; the General Hospital, Birmingham; and Dudley Road Hospital, Birmingham

SUMMARY In an aseptic microbiological assay of folate compounds and their breakdown compounds, using Lactobacillus casei, Streptococcus faecalis, and Pediococcus cerevisiae, 4a-hydroxy-5-methyl4,5,6,7-tetrahydrofolate and 5-methyl-5,8-dihydrofolate were inactive under all conditions to all three organisms and 5-methyl-5,6-dihydrofolate was inactive unless ascorbate was present in the incubation medium, and then only to $L$. casei. 5-Methyltetrahydrofolate was active only for $L$. casei, and activity in purified samples to $S$. faecalis was due to trace amounts of folic acid. Analysis of $S$. faecalis values in the serum in normal subjects and in patients with various disorders showed that levels of 10-formyltetrahydrofolate are raised in coeliac disease, leukaemia, rheumatoid arthritis, and schizophrenia.

5-Methyltetrahydrofolate is readily absorbed by normal human subjects and by patients with pernicious anaemia but poorly absorbed by patients with coeliac disease or leukaemia. 5-Methyl5,6-dihydrofolate was quickly absorbed by normal human subjects, being reflected by a considerably raised level of 5-methyltetrahydrofolate in serum when sodium bicarbonate was given by mouth before the 5-methyl-5,6-dihydrofolate. These higher levels were comparable to those in patients with pernicious anaemia after oral administration of 5-methyl-5,6-dihydrofolate. Oral 5-methyl5,8-dihydrofolate and 4a-hydroxy-5-methyl-tetrahydrofolate did not appear as microbiologically active folates in the serum.

The findings of this study suggest that the availability for biological utilisation of the major dietary folate compounds will depend on the amount of gastric acidity and of ascorbate in the intestinal chyme. Many may be unavailable for metabolic utilization in the body.

The folates present in foodstuffs before degradation are 5-methyltetrahydrofolate and 10-formyltetrahydrofolate and their derivatives. Both compounds are readily oxidised, the rates varying directly with oxygen concentration, temperature, alkalinity, exposure to light, and concentration of cupric and ferric ions (Pearson, 1974; Blair and Pearson, 1974a, 1974b, Blair et al., 1975). 5-Methyltetrahydrofolate is oxidised to 5-methyl-5,6-dihydrofolate, which can then rearrange to 5-methyl-5, 8-dihydrofolate at $\mathrm{pH}$ 4.0 or is oxidised to 4a-hydroxy-5-methyl-4a,5,6,7tetrahydrofolate (Fig. 1) (Larrabee et al., 1961; Donaldson and Keresztesy, 1962; Gupta and Huennekens, 1967; Blair et al., 1975). 10-Formyltetrahydrofolate is oxidised to 10-formylfolate (Robinson, 1971). Consequently there will be a number of folate compounds present in the diet,

Received for publication 26 October 1976 some of which may not be available for biological utilization in the body but may be microbiologically active for $L$. casei, $S$. faecalis, or $P$. cerevisiae.

In this paper we report the availability of the folates derived from 5-methyltetrahydrofolate and their metabolism in normal humans and in some diseases. A further analysis of the $S$. faecalis values in the serum of normal and diseased subjects is reported.

\section{Material and methods}

5-Methyltetrahydrofolate was prepared by the method of Blair and Saunders (1970), 4a-hydroxy-5-methyl4a,5,6,7-tetrahydrofolate as given by Gapski et al. (1971), and 7,8-dihydrofolate acid as described by Futterman (1963).

5-Methyl-5,6-dihydrofolate was prepared by dissolving $400 \mathrm{mg}$ of 5-methyltetrahydrofolate in $200 \mathrm{ml}$ 
<smiles>CN1c2c(nc(N)[nH]c2=O)NC2CC21CN</smiles><smiles>O=C([O-])CCC(NC(=O)c1ccccc1)C(=O)[O-]</smiles>

5- methyltetrahydrofolate

oxidation<smiles>CN1c2c(nc(N)[nH]c2=O)N=CC1CNc1ccc(C(=O)NC(CCC(=O)[O-])C(=O)[O-])cc1</smiles>

5-methyl-5, 6-dihydrofolate<smiles>CN1C2(CN)C(=O)NC(N)=NC2=NC2C(=O)C21O</smiles><smiles>CC</smiles><smiles>CN1C(CNc2ccc(C(=O)NC(CCC(=O)[O-])C(=O)[O-])cc2)=CNc2nc(N)[nH]c(=O)c21</smiles>

5-methyl-5, 8-dihydrofolate

\section{4a-hydroxy-5-methyl-4a, 5, 6,7-tetrahydrofolate}

Fig. 1 5-Methyltetrahydrofolate and its derivatives.

of water containing $5 \mathrm{mg}$ of copper sulphate. The mixture was stirred under a slow stream of oxygen for 60-100 minutes and the reaction progress followed by the appearance of the characteristic ultraviolet spectrum of 5-methyl-5,6-dihydrofolate with maxima at 248 and $290 \mathrm{~nm}$ in $\mathbf{0 \cdot 1} \mathrm{M}$ phosphate buffer $\mathrm{pH} 7 \cdot 0$. The solution of 5-methyl-5,6-dihydrofolate was freeze-dried to yield a puffy yellow solid product of
$90 \%$ purity as judged by ultraviolet spectroscopy. The crude product was then further purified as described by Gapski et al. (1971).

5-Methyl-5,8-dihydrofolate was prepared by acidic rearrangement of 5-methyl-5,6-dihydrofolate. A $5-\mathrm{mg}$ sample of microbiologically active material of 5-methyl-5,6-dihydrofolate was dissolved in $0.1 \mathrm{M}$ $\mathrm{HCl} 30$ minutes before administration to normal 
human volunteers. The resultant acidified samples of 5-methyl-5,8-dihydrofolate were neutralised with sodium hydroxide solution just before use.

MICROBIOLOGICAL ASSAY

Microbiological assays of 5-methyl-5,6-dihydrofolate; 5-methyl-5,8-dihydrofolate; 4a-hydroxy-5methyl-4a,5,6,7-tetrahydrofolate; and of the serum samples were performed aseptically using $L$. casei, $S$. faecalis, and $P$. cerevisiae (Herbert, 1966).

Since 5-methyl-5,6-dihydrofolate is microbiologically active for $L$. casei in the presence of ascorbate but inactive when ascorbate is omitted its presence cannot be detected in serum or plasma with added ascorbate, but it can be determined after its rapid conversion in acid to the microbiologically inactive 5-methyl-5,8-dihydrofolate by the difference in $L$. casei values in their two situations.

Blood samples from normal human volunteers and patients were separated quickly (30 minutes clotting time) to avoid oxidation and the sera divided into two equal portions. The first portion was adjusted to a $\mathrm{pH}$ of 3.0 with concentrated $\mathrm{HCl}$ and allowed to stand in the dark at room temperature for 20 minutes. At the end of the 20-minutes period a small amount of $20 \%(w / v)$ sodium hydroxide solution was added to bring the $\mathrm{pH}$ of the sample to the starting $\mathrm{pH}$ of around 7.4 and ascorbate $(5 \mathrm{mg} / \mathrm{ml})$ added. To the second portion an amount of distilled water was added equal to the volume of added acid and base and ascorbate $(5 \mathrm{mg} / \mathrm{ml})$. These samples were kept at $-20^{\circ} \mathrm{C}$ until assayed microbiologically, which in all cases was within a week.

\section{SERUM VALUES}

Single blood samples were taken after a normal lunch from 52 normal persons and 353 patients with serum folate levels $>3.0 \mu \mathrm{g} / \mathrm{l}$. The patients were suffering from the following: rheumatoid arthritis (25); leukaemia (79); coeliac disease, treated and untreated (74); schizophrenia (7); treated pernicious anaemia (18); psoriasis (6); and regional enteritis (144). Likewise blood samples were taken from 19 normal persons and 190 patients with serum folate levels $<3.0 \mu \mathrm{g} / \mathrm{l}$. The patients were suffering from the following: coeliac disease (30); psoriasis (5); leukaemia (31); untreated pernicious anaemia (2); rheumatoid arthritis (22); regional enteritis (90); and schizophrenia (10).

Fasting blood samples were taken from eight patients with folic acid deficient megaloblastic anaemia.

Fasting blood samples were taken from five normal persons at hourly intervals for 10 hours.

ABSORPTION TESTS

After an overnight fast the serum levels of folate compounds after oral administration of 5-methyltetrahydrofolate were measured in 15 normal volunteers, two patients with untreated pernicious anaemia, six patients with untreated coeliac disease, and eight patients with chronic granulocytic leukaemia. Also the serum levels of 5-methyl-5,6dihydrofolate were measured in five normal volunteers and five patients with untreated pernicious anaemia. The first venous blood samples were taken aseptically just before giving the test material. Further samples were taken while still fasting at $30,60,90,120$, and 180 minutes. Blood was allowed to clot, centrifuged, and ascorbate was added (2-5 $\mathrm{mg} / \mathrm{ml}$ ) to the separated serum and kept at $-20^{\circ} \mathrm{C}$ until assayed.

The serum levels after oral administration of $10 \mathrm{mg}$ 4a-hydroxy-5-methyltetrahydrofolate, 5-methy'-5,8dihydrofolate, and dihydrofolate were similarly studied.

To study the effect of altering the gastric $\mathrm{pH}$ a dose of 3 or $5 \mathrm{~g}$ sodium bicarbonate was administered before additional tests, and to prevent oxidation $1 \mathrm{~g}$ ascorbic acid was similarly given in other experiments.

\section{Results}

\section{MICROBIOLOGICAL ACTIVITY OF FOLATE} COMPOUNDS

Aseptic microbiological assay of 5-methyl-5,6dihydrofolate; 5-methyl-5,8-dihydrofolate; and 4ahydroxy-5-methyl-4,5,6,7-tetrahydrofolate with all three micro-organisms in the presence of ascorbate showed that only the first compound was microbiologically active, and then only for $L$. case $i$, with equivalent activity to that of 5-methyltetrahydrofolate. The other two compounds were inactive for all three micro-organisms. When ascorbate was omitted from the incubation medium 5-methyl-5,6dihydrofolate was also inactive for the three microorganisms.

Bioautography of 1- $\mu \mathrm{g}$ samples of 5-methyltetrahydrofolate in $3 \%$ aqueous ammonium chloride using $L$. case $i$ and $S$. faecalis as developing organisms (Leeming et al., 1970; Brown et al., 1973) showed a little $L$. case $i$ activity (3-10\%) at $R_{F} 0.0$ (folic acid) and much activity at $R_{F} 0.65$ (5-methyltetrahydrofolate) but $S$. faecalis activity only at $R_{F} 0.0$ and none elsewhere - that is, $S$. faecalis activity associated with highly purified samples of 5-methyltetrahydrofolate was due to trace amounts of folic acid.

The difference between the $S$. faecalis and $P$. cerevisiae values was nil at short clotting times (Table 1). The $S$. faecalis value remained constant over six hours while that of $\boldsymbol{P}$. cerevisiae steadily declined from the same as the $S$. faecalis value to 
$60 \%$ of it after eight hours (Table 1 ). Since the only known $P$. cerevisiae-active folate which would rapidly decompose to yield an equivalent and stable $S$. faecalis activity is 10 -formyltetrahydrofolate, this provides further evidence that 10-formyltetrahydrofolate is a folate present in serum. The $S$. faecalis value would therefore seem to be the best measure of 10-formyltetrahydrofolate in the serum of normal subjects. However, when oral doses of other folates are given, folic acid may be present as an impurity, so that levels of 10 -formyltetrahydrofolate are best represented in such experiments by the $P$. cerevisiae values.

\section{SERUM VALUES}

Table 2 compares the serum $S$. faecalis values of normal and diseased subjects whose $L$. casei-active folates ranged from $3.0 \mu \mathrm{g} / 1$ to $20 \mu \mathrm{g} / 1$ in the nonfasting state. Table 3 is a similar comparison of those with serum folates below $3.0 \mu \mathrm{g} / \mathrm{l}$. In many cases the $S$. faecalis level is significantly increased in the diseased state. The diurnal variation experiment showed a varying level of serum folates in man (Fig. 2), and though the differences in levels are not significant the existence of a diurnal variation is established by the smooth curve obtained.

Measurement of the 5-methyltetrahydrofolate component in normal persons in this study showed it to contain a small amount of 5-methyl-5,6-dihydrofolate (Table 4) in some subjects but not in others, suggesting a possible analytical artefact. In pernicious anaemia the increase in the level of serum 5-methyl5,6-dihydrofolate was significant and too large to be an artefact.

Table 1 Effect of clotting time $(\mathrm{T})$ on serum folates (mean $\pm S E M$ of three samples)

\begin{tabular}{|c|c|c|c|c|c|c|c|c|c|}
\hline Test organism & $\mathbf{T}_{\frac{1}{2}}$ & $T_{1}$ & $T_{1 \frac{1}{2}}$ & $\mathbf{T}_{2}$ & $T_{2 \frac{1}{2}}$ & $T_{3}$ & $T_{4}$ & $T_{\text {. }}$ & $T_{8}$ \\
\hline L. casei & $\begin{array}{r}7.0 \\
\pm 0.7\end{array}$ & $\begin{array}{r}7.0 \\
\pm 0.7\end{array}$ & $\begin{array}{r}6.8 \\
\pm 0.7\end{array}$ & $\begin{array}{r}7.0 \\
\pm 0.7\end{array}$ & $\begin{array}{r}7.2 \\
+0.8\end{array}$ & $\begin{array}{r}7.0 \\
+0.8\end{array}$ & $\begin{array}{r}6.7 \\
+0.8\end{array}$ & $\begin{array}{r}6.7 \\
+0.8\end{array}$ & $\begin{array}{r}6.8 \\
+0.8\end{array}$ \\
\hline S. faecalis & $\begin{array}{r}0.8 \\
\pm 0.1\end{array}$ & $\begin{array}{r}0.8 \\
+0.1\end{array}$ & $\begin{array}{r}0.8 \\
+0.1\end{array}$ & $\begin{array}{r}0.8 \\
+0.1\end{array}$ & $\begin{array}{r}0.8 \\
+0.1\end{array}$ & $\begin{array}{r}0.8 \\
+0.1\end{array}$ & $\begin{array}{r} \pm 0.0 \\
0.8 \\
+0.1\end{array}$ & $\begin{array}{r} \pm 0.8 \\
0.8 \\
+0.1\end{array}$ & $\begin{array}{r} \pm 0.8 \\
0.8 \\
+0.1\end{array}$ \\
\hline P. cerevisiae & $\begin{array}{r}0.8 \\
\pm 0.1\end{array}$ & $\begin{array}{r}0.7 \\
\pm 0.1\end{array}$ & $\begin{aligned} & 0.65 \\
& \pm 0.1\end{aligned}$ & $\begin{array}{r}0.6 \\
\pm 0.1\end{array}$ & $\begin{aligned} & 0.65 \\
\pm & 0.1\end{aligned}$ & $\begin{array}{r}0.6 \\
\pm 0.1\end{array}$ & $\begin{array}{r}0.6 \\
\pm 0.1\end{array}$ & $\begin{array}{r}0.6 \\
\pm 0.1\end{array}$ & $\begin{array}{r}0.6 \\
\pm 0.1\end{array}$ \\
\hline
\end{tabular}

$\mathbf{T}=$ time in hours.

Table 2 Serum folate levels as microbiologically assayed with $\mathrm{L}$. casei and $\mathrm{S}$. faecalis of normal subjects and patients with L. casei values of 3.0-20 $\mathrm{g} / \mathrm{l}$. All samples taken in early afternoon after usual diet

\begin{tabular}{|c|c|c|c|c|c|}
\hline Diagnosis & $\begin{array}{l}\text { No. of } \\
\text { subjects }\end{array}$ & $\begin{array}{l}\text { L. casei } \\
(\mu g / l \pm S E M)\end{array}$ & $\begin{array}{l}\text { Student's t test } \\
\text { (P) }\end{array}$ & $\begin{array}{l}\text { S. faecalis } \\
(\mu g / l \pm S E M)\end{array}$ & $\begin{array}{l}\text { Student's t test } \\
\text { (P) }\end{array}$ \\
\hline Normal & 52 & $5 \cdot 57 \pm 0.28$ & - & $0.78 \pm 0.03$ & - \\
\hline Adult coeliac disease & 74 & $6 \cdot 10 \pm 0.40$ & NS & $1.00 \pm 0.06$ & 0.005 \\
\hline Psoriasis & 6 & $4.73 \pm 0.50$ & NS & $0.95 \pm 0.14$ & $0 \cdot 100$ \\
\hline Leukaemia & 79 & $5.56 \pm 0.28$ & NS & $1.65 \pm 0.10$ & 0.001 \\
\hline Pernicious anaemia* & 18 & $6.50 \pm 0.70$ & NS & $0.89 \pm 0.10$ & NS \\
\hline Rheumatoid arthritis & 25 & $4.72 \pm 0.36$ & 0.10 & $1.85 \pm 0.30$ & 0.001 \\
\hline Regional enteritis & 144 & $5 \cdot 29 \pm 0.22$ & NS & $0.84 \pm 0.03$ & NS \\
\hline Schizophrenia & 7 & $3.94 \pm 0.40$ & 0.005 & $0.94 \pm 0.10$ & 0.05 \\
\hline
\end{tabular}

NS = not significant.

* Receiving vitamin $\mathbf{B}_{12}$.

Table 3 Comparison of serum folate levels of normal subjects and patients with $\mathrm{L}$. casei values $<3.0 \mu \mathrm{g} / \mathrm{l}$. All samples taken in early afternoon after usual diet

\begin{tabular}{|c|c|c|c|c|c|}
\hline Diagnosis & $\begin{array}{l}\text { No. of } \\
\text { subjects }\end{array}$ & $\begin{array}{l}\text { L. casei } \\
(\mu g / l \pm S E M)\end{array}$ & $\begin{array}{l}\text { Student's t test } \\
\text { (P) }\end{array}$ & $\begin{array}{l}\text { S. faecalis } \\
(\mu g / l \pm S E M)\end{array}$ & $\begin{array}{l}\text { Student's t test } \\
\text { (P) }\end{array}$ \\
\hline Normal & 19 & $2.00 \pm 0.20$ & - & $0.40 \pm 0.06$ & - \\
\hline Adult coeliac disease & 30 & $1.85 \pm 0.15$ & NS & $0.90 \pm 0.08$ & 0.001 \\
\hline Psoriasis & 5 & $1.72 \pm 0.18$ & NS & $1 \cdot 14 \pm 0.18$ & 0.001 \\
\hline Leukaemia & 31 & $1.67 \pm 0.14$ & $0 \cdot 10$ & $1.03 \pm 0.10$ & 0.001 \\
\hline Pernicious anaemia* & 2 & $1 \cdot 50$ & NS & 0.70 & 0.05 \\
\hline Rheumatoid arthritis & 22 & $2 \cdot 30 \pm 0.10$ & NS & $1 \cdot 29 \pm 0.15$ & 0.001 \\
\hline Regional enteritis & 90 & $1.50 \pm 0.07$ & 0.05 & $0.38 \pm 0.03$ & NS \\
\hline Schizophrenia & 10 & $1.65 \pm 0.18$ & $0 \cdot 10$ & $0.84 \pm 0.10$ & 0.001 \\
\hline
\end{tabular}

* Receiving vitamin $\mathbf{B}_{\mathbf{1 2}}$.

NS = not significant. 


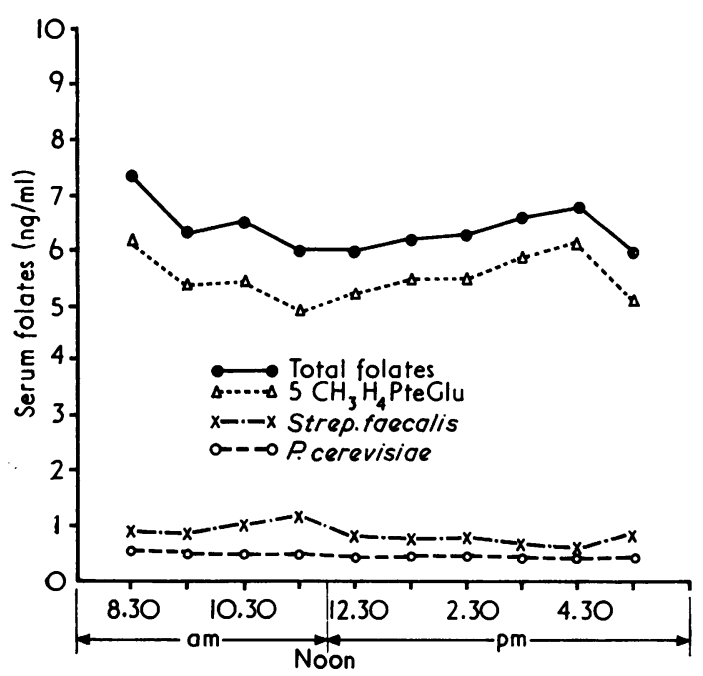

Fig. 2 Diurnal variation of serum folates of fasting normal persons during working day.

Eight patients with megaloblastic anaemia had a mean cell volume of $108 \pm 1.5 \mu \mathrm{m}^{3}$ and serum 10-formyltetrahydrofolate of $0.16 \pm 0.04 \mu \mathrm{g} / \mathrm{l}$, significantly less than that in normal subjects (Tables 2 and 3). Patients with coeliac disease, leukaemia, rheumatoid arthritis, and schizophrenia had significantly increased levels of 10-formyltetrahydrofolate, but those with regional enteritis and pernicious anaemia had levels not significantly different from normal. Patients with psoriasis had significantly raised levels of $\mathbf{1 0 - f o r m y l t e t r a h y d r o f o - ~}$ late when the total serum folate was $<3.0 \mu \mathrm{g} / \mathrm{l}$, but when the serum folate was $>3.0 \mu \mathrm{g} / \mathrm{l}$ the level of 10-formyltetrahydrofolate when the total serum folate was tetrahydrofolate, although raised, was not significantly different from normal.
ABSORPTION TESTS

Microbiologically active 5-methyl-5,6-dihydrofolate $5 \mathrm{mg}$ by mouth quickly appears in the serum (Table 5 ) and at significantly higher levels when given with sodium bicarbonate (Table 6). At the same time 5-methyl-5,6-dihydrofolate is converted at some stage after administration to 5-methyltetrahydrofolate. The resultant serum levels of this latter compound are still further enhanced by the simultaneous administration of sodium bicarbonate, the differences being significant by Student's $t$ test at $60,90,120$, and 180 minutes after ingestion (Table 6) $(P<0.01)$.

Serum levels after the oral administration of 5-methyltetrahydrofolate are shown in Table 7 and also with the simultaneous ingestion of ascorbate (Table 8 ) and of bicarbonate (Tables 9 and 10), when significantly higher serum levels were found. Oral administration of 5-methyl-5,8-dihydrofolate (Table 11) and 4a-hydroxy-5-methyl-4a,5,6,7-tetrahydrofolate (Table 12) showed little rise in folate serum levels. Administration of 7,8-dihydrofolate gave a marked increase in total serum folates but not serum 10-formyltetrahydrofolate (Table 13). Studies in patients with pernicious anaemia after oral 5-methyltetrahydrofolate (Table 14) and after 5-methyl-5,6dihydrofolate (Table 15) showed that significant levels of the relevant folates were readily attained. Oral administration of 5-methyl-5,6-dihydrofolate to patients either with coeliac disease (Table 16) or with leukaemia (Table 17) showed significantly reduced serum levels compared with similar experiments in normal subjects.

\section{Discussion}

Although published information on the serum levels of folate in normal and diseased subjects after oral administration of folic acid is considerable, little is known about the levels after the administration of 5-methyltetrahydrofolate and its decomposition products 5-methyl-5,6-dihydrofolate, 4a-hydroxy-5-

Table 4 Fasting serum 5-methyl-5,6-dihydrofolate levels in normal subjects and those with various diseases

\begin{tabular}{|c|c|c|c|c|c|}
\hline \multirow[t]{2}{*}{ Diagnosis } & \multirow{2}{*}{$\begin{array}{l}\text { No. of } \\
\text { subjects }\end{array}$} & \multicolumn{2}{|l|}{ L. casei $(\mu g / l \pm S E M)$} & \multirow{2}{*}{$\begin{array}{l}\text { 5-CH } \mathrm{CH}_{\mathbf{3}}-\mathbf{5}, 6-\mathrm{H}_{2}-\text { PteGlu } \\
(\mu g / l \pm S E M)\end{array}$} & \multirow{2}{*}{$\begin{array}{l}\text { 5-CH }{ }_{3} \mathbf{H}_{4}-\text { PteGlu* } \\
(\mu g / l \pm S E M)\end{array}$} \\
\hline & & Before acid treatment & After acid treatment & & \\
\hline $\begin{array}{l}\text { Normal } \\
\text { Leukaemia } \\
\text { Regional enteritis } \\
\text { Pernicious anaemia } † \\
\text { Rheumatoid arthritis } \\
\text { Adult coeliac disease }\end{array}$ & $\begin{array}{r}30 \\
5 \\
8 \\
4 \\
30 \\
4\end{array}$ & $\begin{array}{l}5.75 \pm 0.48 \\
5.18 \pm 0.50 \\
4.78 \pm 0.38 \\
9.50 \pm 4.30 \\
3.3 \pm 0.28 \\
6.4 \pm 1.00\end{array}$ & $\begin{array}{l}5.00 \pm 0.63 \\
4.50 \pm 0.93 \\
4.47 \pm 0.63 \\
5.50 \pm 2.90 \\
2.42 \pm 0.44 \\
5.55 \pm 0.50\end{array}$ & $\begin{array}{l}0.75 \pm 0.15 \\
0.68 \pm 0.43 \\
0.31 \pm 0.25 \\
4.00 \pm 1.41 \\
0.88 \pm 0.16 \\
0.85 \pm 0.15\end{array}$ & $\begin{array}{l}4.95 \pm 0.40 \\
4.00 \pm 0.45 \\
4.00 \pm 0.35 \\
8.70 \pm 4.20 \\
1.80 \pm 0.20 \\
5.40 \pm 0.90\end{array}$ \\
\hline
\end{tabular}

*Summation of 5- $\mathrm{CH}_{3} \mathrm{H}_{4}$ PteGlu and $5-\mathrm{CH}_{3}-5,6-\mathrm{H}_{2}$ PteGlu activities.

+Receiving vitamin $\mathbf{B}_{12}$. 
Table 5 Serum folate levels in five normal persons after oral administration of $10 \mathrm{mg}$ of 5-methyl-5,6-dihydrofolate

\begin{tabular}{|c|c|c|c|c|c|c|c|c|}
\hline \multirow[t]{2}{*}{ Serum folate } & \multicolumn{8}{|c|}{ Serum folate levels $(\mu \mathrm{g} / \mathrm{l} \pm S E M)$} \\
\hline & $\mathbf{T}_{0}$ & $T_{1}$ & $\mathbf{T}_{1}$ & $\mathbf{T}_{1 \pm}$ & $\mathrm{T}_{2}$ & $\mathbf{T}_{3}$ & $T_{4}^{*}$ & $T_{0}^{*}$ \\
\hline $\begin{array}{l}\text { Total folates } \\
5-\mathrm{CH}_{3} \mathrm{H}_{4} \text { PteGlu } \dagger \\
5-\mathrm{CH}_{3}-5,6-\mathrm{H}_{2} \text { PteGlu* } \\
\text { 10-CHO } \mathrm{H}_{4} \text { PteGlu } \\
\text { S. faecalis }\end{array}$ & $\begin{array}{l}6 \cdot 2 \pm 1 \cdot 3 \\
5 \cdot 5 \pm 1 \cdot 3 \\
0 \cdot 0 \\
0 \cdot 6 \pm 0 \cdot 2 \\
0 \cdot 7 \pm 0 \cdot 25\end{array}$ & $\begin{array}{l}37.6 \pm 5.9 \\
36.6 \pm 5.9 \\
2.0 \\
0.9 \pm 0.2 \\
1.0 \pm 0.25\end{array}$ & $\begin{array}{l}32 \cdot 8 \pm 4 \cdot 5 \\
31 \cdot 6 \pm 4 \cdot 6 \\
1 \cdot 0 \\
1 \cdot 0 \pm 0 \cdot 2 \\
1.2 \pm 0 \cdot 28\end{array}$ & $\begin{aligned} 27 \cdot 8 & \pm 2 \cdot 0 \\
26 \cdot 8 & \pm 2 \cdot 0 \\
1 \cdot 0 & \\
0 \cdot 9 & \pm 0 \cdot 2 \\
1.0 & \pm 0 \cdot 25\end{aligned}$ & $\begin{aligned} 23 \cdot 6 & \pm 0.9 \\
22 \cdot 7 & \pm 0 \cdot 9 \\
1 \cdot 0 & \\
0.8 & \pm 0 \cdot 2 \\
0.9 & \pm 0.25\end{aligned}$ & $\begin{aligned} 21 \cdot 8 & \pm 0 \cdot 8 \\
21 \cdot 1 & \pm 0 \cdot 2 \\
1 \cdot 0 & \\
0 \cdot 6 & \pm 0 \cdot 1 \\
0 \cdot 7 & \pm 0 \cdot 1\end{aligned}$ & $\begin{array}{r}21 \cdot 0 \\
20 \cdot 0 \\
0.5 \\
1.0 \\
1.0\end{array}$ & $\begin{array}{r}20 \cdot 0 \\
19 \cdot 0 \\
0.5 \\
1.0 \\
1.0\end{array}$ \\
\hline
\end{tabular}

* Results from only one subject

†Summation of 5- $\mathrm{CH}_{3} \mathrm{H}_{4} \mathrm{PteGlu}$ and $5-\mathrm{CH}_{3}-5,6-\mathrm{H}_{2}$ PteGlu activities.

$+P$. cerevisiae values.

$\mathrm{T}=$ time in hours.

Table 6 Serum folate levels in five normal persons after oral administration of $3 \mathrm{~g}$ sodium bicarbonate and 10 mg dl-5-methyl-5,6-dihydrofolate

\begin{tabular}{|c|c|c|c|c|c|c|c|c|}
\hline \multirow[t]{2}{*}{ Serum folate } & \multicolumn{8}{|c|}{ Serum folate levels $(\mu g / l \pm S E M)$} \\
\hline & $\mathbf{T}_{\mathbf{0}}$ & $\mathbf{T}_{\frac{1}{2}}$ & $\mathbf{T}_{1}$ & $\mathbf{T}_{1 \frac{1}{2}}$ & $\mathrm{~T}_{2}$ & $\mathbf{T}_{\mathbf{3}}$ & $T_{4}^{*}$ & $\mathbf{T}_{6} *$ \\
\hline $\begin{array}{l}\text { Total folates } \\
5-\mathrm{CH}_{3} \mathrm{H}_{4} \text { PreGlu } \dagger \\
5-\mathrm{CH}_{3}-5,6-\mathrm{H}_{2} \text { PteGlu* }^{*} \\
10-\mathrm{CHO} \mathrm{H}_{4} \mathrm{PteGlu}_{+}^{+} \\
\text {S. faecalis }\end{array}$ & $\begin{array}{l}8.8 \pm 3.0 \\
8.0 \pm 3.0 \\
0.5 \\
0.7 \pm 0.1 \\
0.8 \pm 0.1\end{array}$ & $\begin{array}{l}19.0 \pm 1.0 \\
18.2 \pm 1.0 \\
11.0 \\
0.8 \pm 0.1 \\
0.8 \pm 0.1\end{array}$ & $\begin{array}{l}51 \cdot 6 \pm 3.4 \\
50.3 \pm 4.0 \\
18.0 \\
1.0 \pm 0.1 \\
1.4 \pm 0.4\end{array}$ & $\begin{array}{r}112 \cdot 3 \pm 10 \cdot 0 \\
109 \cdot 9 \pm 10 \cdot 2 \\
33 \cdot 0 \\
1 \cdot 3 \pm 0 \cdot 2 \\
2 \cdot 4 \pm 0 \cdot 2\end{array}$ & $\begin{array}{l}99.5 \pm 12.0 \\
96.4 \pm 12.4 \\
26.0 \\
1.5 \pm 0.4 \\
2.9 \pm 0.3\end{array}$ & $\begin{array}{r}51 \cdot 3 \pm 6.0 \\
48 \cdot 8 \pm 6.4 \\
10.0 \\
1 \cdot 2 \pm 0.5 \\
2.5 \pm 0.4\end{array}$ & $\begin{array}{l}45 \\
43 \cdot 5 \\
-1 \cdot 0 \\
1 \cdot 5\end{array}$ & $\begin{array}{l}32 \\
30 \cdot 8 \\
- \\
0 \cdot 9 \\
1 \cdot 2\end{array}$ \\
\hline
\end{tabular}

* Results from only one subject.

tSummation of $5-\mathrm{CH}_{3} \mathrm{H}_{4}$ PteGlu and $5-\mathrm{CH}_{3}-5,6-\mathrm{H}_{2}$ PteGlu activities.

$+P$. cerevisiae values.

$\mathbf{T}=$ time in hours.

Table 7 Serum folate levels in six normal persons after oral administration of $10 \mathrm{mg}$ 5-methyltetrahydrofolate

\begin{tabular}{|c|c|c|c|c|c|c|c|c|c|}
\hline \multirow[t]{2}{*}{ Serum folates } & \multicolumn{9}{|c|}{ Serum folate levels $(\mu \mathrm{g} / l \pm S E M)$} \\
\hline & $\mathrm{T}_{0}$ & $\mathrm{~T}_{\frac{1}{2}}$ & $\mathbf{T}_{1}$ & $\mathbf{T}_{1 \frac{1}{2}}$ & $\mathbf{T}_{2}$ & $\mathbf{T}_{3}$ & $\mathbf{T}_{4}$ & $T_{5}$ & $T_{0}^{*}$ \\
\hline $\begin{array}{l}\text { Total folates } \\
\text { 5- } \mathrm{CH}_{3} \mathrm{H}_{4} \mathrm{PteGlu} \\
\text { 10-CHOH } \\
\text { S. faecalis }\end{array}$ & $\begin{array}{l}9 \cdot 1 \pm 2 \cdot 1 \\
8 \cdot 3 \pm 2 \cdot 3 \\
0 \cdot 6 \pm 0 \cdot 1 \\
0 \cdot 8 \pm 0 \cdot 15\end{array}$ & $\begin{array}{c}106 \cdot 0 \pm 15 \cdot 2 \\
103 \cdot 1 \pm 16 \cdot 0 \\
0.5 \pm 0 \cdot 1 \\
2.9 \pm 0.6\end{array}$ & $\begin{array}{c}166.0 \pm 21.0 \\
162.0 \pm 21.6 \\
0.7 \pm 0.1 \\
3.8 \pm 0.6\end{array}$ & $\begin{array}{c}188.0 \pm 19.0 \\
184.3 \pm 21.0 \\
0.6 \pm 0.1 \\
3.7 \pm 0.6\end{array}$ & $\begin{array}{c}180.0 \pm 14.7 \\
175.4 \pm 14.7 \\
0.8 \pm 0.09 \\
4.6 \pm 0.6\end{array}$ & $\begin{aligned} 110.0 & \pm 9.4 \\
106.3 & \pm 9.4 \\
0.7 & \pm 0.09 \\
3.7 & \pm 0.5\end{aligned}$ & $\begin{array}{c}81.0 \pm 9.8 \\
77.5 \pm 10.6 \\
0.7 \pm 0.1 \\
3.5 \pm 0.5\end{array}$ & $\begin{array}{c}55 \cdot 8 \pm 5 \cdot 2 \\
52 \cdot 4 \pm 5 \cdot 8 \\
0 \cdot 7 \pm 0 \cdot 1 \\
3 \cdot 4 \pm 0.6\end{array}$ & $\begin{array}{r}41 \cdot 0 \\
37 \cdot 8 \\
0 \cdot 7 \\
3 \cdot 2\end{array}$ \\
\hline
\end{tabular}

* Results from only one subject.

+Summation of $5-\mathrm{CH}_{3} \mathrm{H}_{4}$ PteGlu and $5-\mathrm{CH}_{3}-5,6-\mathrm{H}_{2}$ PteGlu activities.

$¥ P$. cerevisiae values.

$T=$ time in hours.

Table 8 Serum folate levels in three normal persons after oral administration of $20 \mathrm{mg}$ 5-methyltetrahydrofolate in $200 \mathrm{ml}$ water containing $1 \mathrm{~g}$ ascorbic acid

\begin{tabular}{|c|c|c|c|c|c|c|}
\hline \multirow[t]{2}{*}{ Serum folate } & \multicolumn{6}{|c|}{ Serum folate levels $(\mu g / l \pm S E M)$} \\
\hline & $\mathbf{T}_{0}$ & $\mathbf{T}_{\frac{1}{2}}$ & $\mathbf{T}_{\mathbf{1}}$ & $T_{1 \frac{1}{2}}$ & $\mathbf{T}_{2}$ & $T_{3}$ \\
\hline $\begin{array}{l}\text { Total folates } \\
5-\mathrm{CH}_{3} \mathrm{H}_{4} \text { PteGlu* } \\
5-\mathrm{CH}_{3}-5,6-\mathrm{H}_{2} \text { PteGlu } \\
\text { 10-CHO } \mathrm{H}_{4} \text { PteGlu } \dagger \\
\text { S. faecalis }\end{array}$ & $\begin{array}{l}9.75 \pm 0.8 \\
9.05 \pm 0.8 \\
0.0 \pm 0.0 \\
0.53 \pm 0.05 \\
0.7 \pm 0.0\end{array}$ & $\begin{array}{c}251.2 \pm 43.0 \\
243.0 \pm 41.0 \\
25.0 \pm 10.4 \\
1.68 \pm 0.45 \\
8.2 \pm 2.5\end{array}$ & $\begin{aligned} & 450 \cdot 0 \pm 23 \cdot 8 \\
& 436 \cdot 4 \pm 17 \cdot 0 \\
& 60 \cdot 0 \pm 17 \cdot 3 \\
& 3 \cdot 25 \pm 0 \cdot 52 \\
& 13 \cdot 6 \pm 5 \cdot 2\end{aligned}$ & $\begin{aligned} & 620 \cdot 0 \pm 47 \cdot 8 \\
& 602 \cdot 2 \pm 42 \cdot 0 \\
& 56 \cdot 7 \pm 17 \cdot 6 \\
& 3 \cdot 32 \pm 0 \cdot 58 \\
& 17 \cdot 8 \pm 6.2\end{aligned}$ & $\begin{array}{r}530.0 \pm 66 \cdot 6 \\
511 \cdot 2 \pm 60 \cdot 0 \\
56.7 \pm 17.6 \\
4 \cdot 35 \pm 0.30 \\
18.8 \pm 7.0\end{array}$ & $\begin{array}{c}430 \cdot 0 \pm 47 \cdot 2 \\
413 \cdot 4 \pm 42 \cdot 0 \\
40 \cdot 0 \pm 15 \cdot 3 \\
4 \cdot 0 \pm 0 \cdot 4 \\
16 \cdot 6 \pm 6 \cdot 1\end{array}$ \\
\hline
\end{tabular}

*Summation of 5- $\mathrm{CH}_{3} \mathrm{H}_{4}$ PteGlu and $5-\mathrm{CH}_{3}-5,6-\mathrm{H}_{2}$ PteGlu activities.

$+P$. cerevisiae values.

$\mathbf{T}=$ time in hours. 
Table 9 Serum folate levels in four normal persons after oral administration of $3 \mathrm{~g}$ of sodium bicarbonate and $10 \mathrm{mg}$ of 5-methyltetrahydrofolate

\begin{tabular}{|c|c|c|c|c|c|c|}
\hline \multirow[t]{2}{*}{ Serum folate } & \multicolumn{6}{|c|}{ Serum folate levels $(\mu g / l \pm S E M)$} \\
\hline & $\mathbf{T}_{0}$ & $\mathbf{T}_{\frac{1}{2}}$ & $T_{1}$ & $T_{1 \frac{1}{2}}$ & $\mathbf{T}_{2}$ & $\mathrm{~T}_{3}$ \\
\hline $\begin{array}{l}\text { Total folates } \\
5-\mathrm{CH}_{3} \mathrm{H}_{4} \text { PteGlu* } \\
5-\mathrm{CH}_{3}-5,6-\mathrm{H}_{2} \mathrm{PteGlu} \\
\text { 10-CHO } \mathrm{H}_{4} \text { PteGlu } \\
\text { S. faecalis }\end{array}$ & $\begin{array}{l}7 \cdot 5 \pm 2 \cdot 2 \\
7 \cdot 0 \pm 2 \cdot 0 \\
3 \cdot 0 \pm 2 \cdot 1 \\
0.5 \pm 0 \cdot 1 \\
0.5 \pm 0 \cdot 1\end{array}$ & $\begin{array}{r}75.0 \pm 7.6 \\
72.2 \pm 7.9 \\
17.0 \pm 2.7 \\
1.0 \pm 0.3 \\
2.8 \pm 1.9\end{array}$ & $\begin{aligned} 155 \cdot 0 & \pm 21 \cdot 8 \\
149 \cdot 6 & \pm 22 \cdot 0 \\
48 \cdot 0 & \pm 17 \cdot 3 \\
1 \cdot 5 & \pm 0 \cdot 3 \\
5 \cdot 4 & \pm 2 \cdot 3\end{aligned}$ & $\begin{aligned} 176 \cdot 7 & \pm 24 \cdot 8 \\
170 \cdot 0 & \pm 25 \cdot 0 \\
74 \cdot 0 & \pm 40 \cdot 0 \\
1 \cdot 7 & \pm 0 \cdot 4 \\
6 \cdot 7 & \pm 2 \cdot 5\end{aligned}$ & $\begin{array}{c}147 \cdot 5 \pm 28 \cdot 7 \\
141 \cdot 0 \pm 29 \cdot 0 \\
65 \cdot 0 \pm 31 \cdot 5 \\
1.5 \pm 0.5 \\
6.5 \pm 3.5\end{array}$ & $\begin{array}{c}106.0 \pm 29.0 \\
101.5 \pm 29.9 \\
50.0 \pm 16.7 \\
1.4 \pm 0.5 \\
4.5 \pm 2.0\end{array}$ \\
\hline
\end{tabular}

*Summation of $5-\mathrm{CH}_{3} \mathrm{H}_{4} \mathrm{PteGlu}$ and $5-\mathrm{CH}_{3}-5,6-\mathrm{H}_{2}$ PteGlu activities.

$+P$. cerevisiae values.

$\mathbf{T}=$ time in hours.

Table 10 Serum folate levels in five normal persons after oral administration of $5 \mathrm{~g}$ sodium bicarbonate and $10 \mathrm{mg}$ of 5-methyltetrahydrofolate

\begin{tabular}{|c|c|c|c|c|c|c|}
\hline \multirow[t]{2}{*}{ Serum folate } & \multicolumn{6}{|c|}{ Serum folate levels $(\mu g / l \pm S E M)$} \\
\hline & $\mathbf{T}_{\mathbf{0}}$ & $T_{\frac{1}{2}}$ & $\mathbf{T}_{1}$ & $T_{1 \frac{1}{2}}$ & $\mathbf{T}_{2}$ & $\mathbf{T}_{3}$ \\
\hline $\begin{array}{l}\text { Total folates } \\
5-\mathrm{CH}_{3} \mathrm{H}_{4} \text { PteGlu* } \\
5-\mathrm{CH}_{3}-5,6-\mathrm{H}_{2} \mathrm{PteGlu} \\
\text { 10-CHO } \mathrm{H}_{4} \text { PteGlu } \dagger\end{array}$ & $\begin{array}{c}8.0 \pm 3.0 \\
7.3 \pm 3.1 \\
0.9 \pm 0.3 \\
0.65 \pm 0.05\end{array}$ & $\begin{array}{r}172 \cdot 0 \pm 18 \cdot 8 \\
170 \cdot 1 \pm 19 \cdot 0 \\
70.0 \pm 28 \cdot 8 \\
1.46 \pm 0.46\end{array}$ & $\begin{array}{r}246.0 \pm 19 \cdot 4 \\
242 \cdot 8 \pm 19 \cdot 7 \\
86.0 \pm 40.7 \\
2 \cdot 20 \pm 0.53\end{array}$ & $\begin{array}{r}259.0 \pm 18.5 \\
255.3 \pm 18.8 \\
115.4 \pm 43.0 \\
1.90 \pm 0.54\end{array}$ & $\begin{array}{r}220 \cdot 0 \pm 22 \cdot 6 \\
217 \cdot 3 \pm 22 \cdot 9 \\
75 \cdot 0 \pm 31 \cdot 7 \\
1 \cdot 56 \pm 0.36\end{array}$ & $\begin{array}{r}145 \cdot 0 \pm 23 \cdot 2 \\
143 \cdot 4 \pm 23 \cdot 5 \\
54 \cdot 0 \pm 28 \cdot 2 \\
1 \cdot 10 \pm 0 \cdot 22\end{array}$ \\
\hline
\end{tabular}

*Summation of $5-\mathrm{CH}_{3} \mathrm{H}_{4} \mathrm{PteGlu}$ and $5-\mathrm{CH}_{3}-5,6-\mathrm{H}_{2}$ PteGlu activities.

$+P$. cerevisiae values.

$\mathbf{T}=$ time in hours.

Table 11 Serum folate levels in three normal persons after oral administration of $10 \mathrm{mg}$ 5-methyl-5,8-dihydrofolate

\begin{tabular}{|c|c|c|c|c|c|c|}
\hline \multirow[t]{2}{*}{ Serum folate } & \multicolumn{6}{|c|}{ Serum folate levels $(u g / l \pm S E M)$} \\
\hline & $T_{0}$ & $\mathrm{~T}_{\frac{1}{2}}$ & $T_{1}$ & $T_{1 \frac{1}{2}}$ & $\mathbf{T}_{2}$ & $\mathbf{T}_{\mathbf{3}}$ \\
\hline $\begin{array}{l}\text { Total folates } \\
5-\mathrm{CH}_{3} \mathrm{H}_{4} \mathrm{PteGlu}^{*} \\
\text { 10-CHO } \mathrm{H}_{4} \mathrm{PteGlu} \dagger \\
\text { S. faecalis }\end{array}$ & $\begin{array}{l}7 \cdot 3 \pm 3.0 \\
6.8 \pm 3 \cdot 0 \\
0.5 \pm 0.1 \\
0.5 \pm 0.1\end{array}$ & $\begin{array}{l}7 \cdot 7 \pm 2 \cdot 7 \\
7 \cdot 0 \pm 2 \cdot 8 \\
0 \cdot 6 \pm 0 \cdot 1 \\
0.7 \pm 0 \cdot 1\end{array}$ & $\begin{array}{l}8 \cdot 2 \pm 2 \cdot 7 \\
7 \cdot 3 \pm 2 \cdot 8 \\
0 \cdot 6 \pm 0 \cdot 1 \\
0.9 \pm 0 \cdot 1\end{array}$ & $\begin{array}{l}8 \cdot 3 \pm 2 \cdot 8 \\
7 \cdot 5 \pm 2 \cdot 7 \\
0 \cdot 5 \pm 0 \cdot 1 \\
0 \cdot 8 \pm 0 \cdot 2\end{array}$ & $\begin{array}{l}7 \cdot 9 \pm 3 \cdot 1 \\
7 \cdot 3 \pm 3 \cdot 0 \\
0.5 \pm 0 \cdot 1 \\
0.6 \pm 0.2\end{array}$ & $\begin{array}{l}7 \cdot 2 \pm 3.6 \\
6.6 \pm 3.5 \\
0.5 \pm 0.1 \\
0.6 \pm 0.2\end{array}$ \\
\hline
\end{tabular}

*Summation of $5-\mathrm{CH}_{3} \mathrm{H}_{4} \mathrm{PteGlu}$ and $5-\mathrm{CH}_{3}-5,6-\mathrm{H}_{2}$ PteGlu activities.

$+P$. cerevisiae values.

$\mathbf{T}=$ time in hours.

Table 12 Serum folate levels in three normal persons after oral administration of $10 \mathrm{mg} 4$-hydroxy-5-methyl4a,5,6,7-tetra-hydrofolate

\begin{tabular}{|c|c|c|c|c|c|c|}
\hline \multirow[t]{2}{*}{ Serum folate } & \multicolumn{6}{|c|}{ Serum folate levels $(\mu \mathrm{g} / \mathrm{l} \pm S E M)$} \\
\hline & $\mathbf{T}_{0}$ & $T_{\frac{1}{2}}$ & $T_{1}$ & $T_{1 \frac{1}{2}}$ & $\mathrm{~T}_{2}$ & $\mathbf{T}_{3}$ \\
\hline $\begin{array}{l}\text { Total folates } \\
5-\mathrm{CH}_{3} \mathrm{H}_{4} \mathrm{PteGlu}^{*} \\
\text { 10-CHO } \mathrm{H}_{4} \mathrm{PteGlu}^{-} \\
\text {S. faecalis }\end{array}$ & $\begin{array}{r}11.8 \pm 6.7 \\
11.2 \pm 6.7 \\
0.6 \pm 0.0 \\
0.6 \pm 0.0\end{array}$ & $\begin{array}{r}11.5 \pm 6.6 \\
10.8 \pm 6.7 \\
0.6 \pm 0.0 \\
0.7 \pm 0.1\end{array}$ & $\begin{array}{c}10.8 \pm 6.0 \\
10.1 \pm 6.0 \\
0.6 \pm 0.0 \\
0.7 \pm 0.1\end{array}$ & $\begin{array}{r}10.6 \pm 7.0 \\
10.0 \pm 7.0 \\
0.6 \pm 0.0 \\
0.6 \pm 0.0\end{array}$ & $\begin{array}{r}10.8 \pm 7.1 \\
10.2 \pm 7.1 \\
0.6 \pm 0.0 \\
0.6 \pm 0.0\end{array}$ & $\begin{aligned} 11 \cdot 8 & \pm 7 \cdot 3 \\
11 \cdot 2 & \pm 7 \cdot 3 \\
0.6 & \pm 0.0 \\
0.6 & \pm 0.0\end{aligned}$ \\
\hline
\end{tabular}

*Summation of $5-\mathrm{CH}_{3} \mathrm{H}_{4} \mathrm{PteGlu}$ and $5-\mathrm{CH}_{3}-5,6-\mathrm{H}_{2} \mathrm{PteGlu}$ activities.

$+P$. cerevisiae values.

$\mathbf{T}=$ time in hours.

methyl-4a,5,6,7-tetrahydrofolate, and 5-methyl-5,8dihydrofolate.

The results of oral administration of 5-methyltetrahydrofolate vary. When exposed to the acid contents of the normal fasting human stomach a substantial proportion will be converted to 5-methyl5,8-dihydrofolate by oxidation and acid-catalysed rearrangement. Not surprisingly, therefore, the serum levels of 5-methyltetrahydrofolate in normal persons are much lower than when the administration 
Table 13 Serum folate levels in four normal persons after oral administration of $5 \mathrm{mg}$ of 7,8-dihydrofolate

\begin{tabular}{|c|c|c|c|c|c|c|}
\hline \multirow[t]{2}{*}{ Serum folate } & \multicolumn{6}{|c|}{ Serum folate levels $(\mu g / l \pm S E M)$} \\
\hline & $\mathbf{T}_{\mathbf{0}}$ & $\mathbf{T}_{\frac{1}{2}}$ & $T_{1}$ & $T_{1 \xi}$ & $T_{2}$ & $T_{2}$ \\
\hline $\begin{array}{l}\text { 5- } \mathrm{CH}_{2} \mathrm{H}_{4} \text { PteGlu } \\
\text { 5-CH, } \mathrm{CH}_{2}-5,6-\mathrm{H}_{2} \text { PteGlu* } \\
\text { 10-CHO H,PteGlu } \\
\text { 10-CHOPteGlu }\end{array}$ & $\begin{array}{l}5.9 \pm 0.7 \\
0 \\
0.60 \pm 0.03 \\
0.10 \pm 0.03\end{array}$ & $\begin{array}{l}132.0 \pm 18 \cdot 8 \\
0 \\
1 \cdot 50 \pm 0.09 \\
1 \cdot 10 \pm 0.10\end{array}$ & $\begin{array}{c}200 \cdot 0 \pm 28.6 \\
0 \\
1 \cdot 80 \pm 0.21 \\
2^{\prime} 60 \pm 0.30\end{array}$ & $\begin{array}{c}260.0 \pm 27 \cdot 4 \\
0 \\
1 \cdot 80 \pm 0.15 \\
1 \cdot 20 \pm 0.10\end{array}$ & $\begin{array}{c}210.0 \pm 22.7 \\
0 \\
1.50 \pm 0.23 \\
1 \cdot 50 \pm 0.10\end{array}$ & $\begin{array}{c}122.5 \pm 20.9 \\
0 \\
1 \cdot 10 \pm 0.19 \\
0.10 \pm 0.10\end{array}$ \\
\hline
\end{tabular}

* Mean of only two subjects.

$\dagger P$. cerevisiae activity.

$\ddagger$ Difference between $S$. faecalis and $P$. cerevisiae activities.

$\mathbf{T}=$ time in hours.

Table 14 Serum folate levels in two patients with pernicious anaemia after oral administration of $10 \mathrm{mg}$ of 5-methyltetrahydrofolate

\begin{tabular}{|c|c|c|c|c|c|c|}
\hline \multirow[t]{2}{*}{ Serum folate } & \multicolumn{6}{|c|}{ Serum folate levels $(\mu g / l \pm S E M)$} \\
\hline & $\mathbf{T}_{\mathbf{0}}$ & $\mathbf{T}_{\mathbf{z}}$ & $\mathbf{T}_{\mathbf{1}}$ & $\mathbf{T}_{1 \frac{1}{2}}$ & $T_{3}$ & $\mathbf{T}_{\mathbf{3}}$ \\
\hline 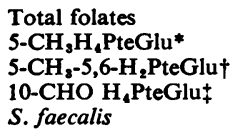 & $\begin{array}{l}3 \cdot 8 \\
3 \cdot 4 \\
1 \cdot 7 \\
0 \cdot 3 \\
0 \cdot 4\end{array}$ & $\begin{array}{r}92 \cdot 0 \\
91 \cdot 5 \\
20.0 \\
0.4 \\
0.5\end{array}$ & $\begin{array}{r}190.0 \\
188.6 \\
70.0 \\
0.6 \\
1.4\end{array}$ & $\begin{array}{r}240.0 \\
238 \cdot 7 \\
120 \cdot 0 \\
0.5 \\
1.3\end{array}$ & $\begin{array}{r}220.0 \\
218.7 \\
70.0 \\
0.9 \\
1.3\end{array}$ & $\begin{array}{r}160 \cdot 0 \\
158 \cdot 7 \\
20 \cdot 0 \\
0 \cdot 8 \\
1 \cdot 3\end{array}$ \\
\hline
\end{tabular}

*Summation of 5- $\mathrm{CH}_{3} \mathrm{H}{ }_{4} \mathrm{PteGlu}$ and $5-\mathrm{CH}_{3}-5,6-\mathrm{H}_{2} \mathrm{PteGlu}$ activities.

$\dagger$ Results from only one subject.

$\ddagger P$. cerevisiae values.

$\mathbf{T}=$ time in hours.

Table 15 Serum folate levels in five patients with pernicious anaemia after oral administration of $10 \mathrm{mg}$ of 5-methyl-5,6-dihydrofolate

\begin{tabular}{|c|c|c|c|c|c|c|}
\hline \multirow[t]{2}{*}{ Serum folate } & \multicolumn{6}{|c|}{ Serum folate levels $(\mu \mathrm{g} / l \pm S E M)$} \\
\hline & $\mathbf{T}_{\mathbf{0}}$ & $T_{\frac{1}{2}}$ & $T_{1}$ & $T_{1 \frac{1}{2}}$ & $\mathbf{T}_{\mathbf{2}}$ & $\mathbf{T}_{\mathbf{3}}$ \\
\hline $\begin{array}{l}\text { Total folates } \\
\text { 5- } \mathrm{CH}_{3} \mathrm{H}_{4} \mathrm{PteGlu}^{*} \\
\text { 5- } \mathrm{CH}_{3}-5,6-\mathrm{H}_{2} \text { PteGlu† } \\
\text { 10-CHO } \mathrm{H}_{4} \text { PteGlu } \\
\text { S. faecalis }\end{array}$ & $\begin{array}{c}8.6 \pm 2.8 \\
7.9 \pm 2.8 \\
4.6 \pm 1.8 \\
0.56 \pm 0.08 \\
0.7 \pm 0.1\end{array}$ & $\begin{array}{c}47 \cdot 0 \pm 6 \cdot 8 \\
45 \cdot 8 \pm 6 \cdot 8 \\
32 \cdot 0 \pm 17 \cdot 5 \\
0 \cdot 73 \pm 0 \cdot 14 \\
1 \cdot 2 \pm 0 \cdot 2\end{array}$ & $\begin{array}{c}72 \cdot 4 \pm 10 \cdot 2 \\
70 \cdot 8 \pm 10 \cdot 1 \\
65 \cdot 0 \pm 22 \cdot 0 \\
0 \cdot 95 \pm 0 \cdot 14 \\
1 \cdot 6 \pm 0.2\end{array}$ & $\begin{array}{r}96.8 \pm 14.1 \\
95.1 \pm 13.9 \\
79.0 \pm 34.0 \\
0.86 \pm 0.12 \\
1.7 \pm 0.25\end{array}$ & $\begin{array}{c}97.4 \pm 15.0 \\
95.7 \pm 14.7 \\
70.0 \pm 26.2 \\
0.85 \pm 0.06 \\
1.7 \pm 0.3\end{array}$ & $\begin{array}{c}84.8 \pm 15.9 \\
83.6 \pm 15.9 \\
47.0 \pm 16 \cdot 1 \\
0.73 \pm 0.09 \\
1.2 \pm 0.2\end{array}$ \\
\hline
\end{tabular}

*Summation of 5- $\mathrm{CH}_{3} \mathrm{H}_{4} \mathrm{PteGlu}$ and $5-\mathrm{CH}_{\mathrm{z}}-5,6-\mathrm{H}_{2} \mathrm{PteGlu}$ activities.

+ Results from three subjects.

$\ddagger P$. cerevisiae values.

$\mathbf{T}=$ time in hours.

Table 16 Serum folate levels in six adults with untreated coeliac disease after oral administration of $10 \mathrm{mg}$ of 5-methyltetrahydrofolate

\begin{tabular}{|c|c|c|c|c|c|c|}
\hline \multirow[t]{2}{*}{ Serum folate } & \multicolumn{6}{|c|}{ Serum folate levels $(\mu g / l \pm S E M)$} \\
\hline & $\mathbf{T}_{\bullet}$ & $\mathbf{T}_{\frac{1}{2}}$ & $\mathbf{T}_{1}$ & $\mathbf{T}_{1 \frac{1}{2}}$ & $\mathbf{T}_{\mathbf{2}}$ & $\mathbf{T}_{\mathbf{2}}$ \\
\hline $\begin{array}{l}\text { Total folates } \\
\text { 5-CH, }{ }_{3} \mathrm{H}_{4} \text { PteGlu* } \\
\text { 5-CH }{ }_{3}-5,6-\mathrm{H}_{2} \text { PteGlu† } \\
\text { 10-CHO H,PteGluł } \\
\text { S. faecalis }\end{array}$ & $\begin{array}{c}4.0 \pm 0.8 \\
3.2 \pm 0.7 \\
0.85 \pm 0.5 \\
0.6 \pm 0.13 \\
0.8 \pm 0.1\end{array}$ & $\begin{array}{r}65.4 \pm 23.9 \\
61.4 \pm 22 \cdot 1 \\
16.6 \pm 16.0 \\
1.0 \pm 0.24 \\
4.0 \pm 1.75\end{array}$ & $\begin{array}{l}96 \cdot 9 \pm 24 \cdot 3 \\
89 \cdot 6 \pm 21 \cdot 2 \\
20 \cdot 0 \pm 15 \cdot 0 \\
1.4 \pm 0.51 \\
7 \cdot 0 \pm 3.1\end{array}$ & $\begin{array}{c}138.0 \pm 25.4 \\
128.2 \pm 21.0 \\
20.0 \pm 15.0 \\
1.8 \pm 0.88 \\
9.8 \pm 4.4\end{array}$ & $\begin{array}{r}132.0 \pm 15.7 \\
121 \cdot 8 \pm 10.5 \\
33 \cdot 7 \pm 24.0 \\
2 \cdot 2 \pm 1 \cdot 19 \\
10.2 \pm 5.3\end{array}$ & $\begin{array}{r}100.0 \pm 20.0 \\
92.6 \pm 16.5 \\
36.7 \pm 20.0 \\
1.2 \pm 0.78 \\
7.4 \pm 3.48\end{array}$ \\
\hline
\end{tabular}

*Summation of 5- $\mathrm{CH}_{2} \mathrm{H}_{4} \mathrm{PteGlu}$ and $5-\mathrm{CH}_{2}-5,6-\mathrm{H}_{2} \mathrm{PteGlu}$ activities.

†Results from three subjects.

$\ddagger P$. cerevisiae values.

$\mathbf{T}=$ time in hours. 
Table 17 Serum folate levels in eight patients with leukaemia after oral administration of $10 \mathrm{mg}$ of 5-methyltetrahydrofolate

\begin{tabular}{|c|c|c|c|c|c|}
\hline \multirow[t]{2}{*}{ Serum folate } & \multicolumn{5}{|c|}{ Serum folate levels $(\mu g / l \pm S E M)$} \\
\hline & $\mathbf{T}_{\mathbf{0}}$ & $\mathbf{T}_{\frac{1}{2}}$ & $\mathbf{T}_{1}$ & $T_{1 \frac{1}{2}}$ & $\mathbf{T}_{\mathbf{2}}$ \\
\hline $\begin{array}{l}\text { Total folates } \\
\text { 5-CH }{ }_{2} H_{4} \text { PteGlu* } \\
\text { 5-CH }{ }_{3}-5,6-H_{2} \text { PteGlu } \dagger \\
\text { 10-CHO H,PteGlu† } \\
\text { S. faecalis }\end{array}$ & $\begin{array}{l}5.7 \pm 1.1 \\
4.8 \pm 1.0 \\
2.5 \\
0.8 \pm 0.05 \\
0.9 \pm 0.08\end{array}$ & $\begin{array}{l}65.6 \pm 12.6 \\
64.6 \pm 12.6 \\
35.0 \\
0.8 \pm 0.07 \\
1.0 \pm 0.09\end{array}$ & $\begin{array}{r}117.9 \pm 19 \cdot 7 \\
116.4 \pm 19.5 \\
23 \cdot 0 \\
1 \cdot 0 \pm 0.17 \\
1 \cdot 5 \pm 0.29\end{array}$ & $\begin{array}{r}105 \cdot 3 \pm 13 \cdot 8 \\
103 \cdot 8 \pm 13 \cdot 8 \\
27 \cdot 0 \\
1 \cdot 0 \pm 0.13 \\
1 \cdot 5 \pm 0.39\end{array}$ & $\begin{array}{l}94.8 \pm 12.7 \\
93.0 \pm 12.7 \\
45.0 \\
1.0 \pm 0.16 \\
1.8 \pm 0.54\end{array}$ \\
\hline
\end{tabular}

*Summation of $5-\mathrm{CH}_{3} \mathrm{H}_{4}$ PteGlu and $5-\mathrm{CH}_{3}-5,6-\mathrm{H}_{2}$ PteGlu activities.

$\dagger$ Results from two subjects.

$+P$. cerevisiae values.

$\mathbf{T}=$ time in hours.

is accompanied by either ascorbate or sodium bicarbonate (Tables 7, 8, 9, 10). Thus these findings reflect chemical changes within the stomach and not necessarily differences in absorption. They contrast with the findings with folic acid, a synthetic compound, where the processes of absorption are depressed (Benn et al., 1971).

Administration of 5-methyltetrahydrofolate to patients with pernicious anaemia resulted in total serum folate levels greater than those in normal subjects as might be deduced from the absence of gastric acidity (Table 14). Weir et al. (1973) found that the absorption of 5-methyltetrahydrofolate was reduced in coeliac disease, and the serum levels in our absorptive experiments in coeliac disease (Table 16) were significantly less than in normal subjects. Similarly, significantly lower serum values were found in the patients with leukaemia (Table 17).

Using the serum levels and the appropriate logarithmic plot the half life of serum folate was $2 \cdot 2$ hours for normal subjects and 3.0 hours for coeliac disease, pernicious anaemia, and leukaemia, the rate of clearance of serum folate being of first order. Hence in the absorptive tests in this paper the serum levels will maintain a relationship to the rate of absorption and thus reflect absorptive ability.

When 5-methyl-5,6-dihydrofolate is given orally to normal subjects, there is a rapid rise in serum folate which reaches a maximum (Table 5) earlier and at much lower levels than that found with the equivalent dose of 5-methyltetrahydrofolate (Table 7), explained most likely by the acid decomposition in the stomach of 5-methyl-5,6-dihydrofolate. This suggestion receives support by the findings that when 5-methyl5,6-dihydrofolate is administered with sodium bicarbonate to normal subjects or in patients with a histamine fast achlorhydria, such as pernicious anaemia, serum folate reaches much higher peak values at the normally expected time (Tables 6 and 15).

Since 4a-hydroxy-5-methyl-4a,5,6,7-tetrahydro- folate is readily absorbed by the rat and excreted unchanged in the urine (Dransfield, 1973), it is likely to be absorbed in man even though the administration of this compound and of 5-methyl-5,8-dihydrofolate produced no change in serum folate (Tables 8 and 9). These two compounds probably do not enter the folate metabolic pool.

It has been suggested (Ratanasthien et al, 1974) that only two folates, 5-methyltetrahydrofolate and 10-formyl-tetrahydrofolate, are present in serum and that the difference observed between the $S$. faecalis and $\boldsymbol{P}$. cerevisiae serum assays in normals is due to the oxidation of 10 -formyltetrahydrofolate to 10 formylfolic acid. The results of the bioautographic analysis of purified 5-methyltetrahydrofolate clearly show that the weak activity of this preparation for $S$. faecalis is due to trace amounts of folic acid.

The results obtained measuring $S$. faecalis and $P$. cerevisiae values over a range of clotting times with the steady decline in the $P$. cerevisiae values (Table 1 ) can only indicate that the relevant folate is 10 formyltetrahydrofolate and the changes in values are due to oxidation to 10 -formylfolic acid. Of the two other possible folates with identical $S$. faecalis and $P$. cerevisiae activity, one, 5-formyltetrahydrofolate is stable to oxidation and the other, tetrahydrofolate, is rapidly oxidised to inactive compounds (Blair and Pearson, 1974a; Pearson, 1974). Consequently, measurement of the levels of 10-formyltetrahydrofolate in the serum (in the absence of administered folic acid) is best determined by the $S$. faecalis value. Interestingly, DaCosta and Rothenberg (1971), using a radioimmune technique, showed that extract human serum contained small amounts of a folate $(1 \cdot 4 \pm 0 \cdot 1$ $\mu \mathrm{g} / \mathrm{l})$ whose properties suggested it was derived from 10-formyltetrahydrofolate.

A comparison of the serum total folate levels in normal subjects and patients with serum levels of $<3 \mu \mathrm{g} / \mathrm{l}$ (Table 3) and in those with levels $>3 \mu \mathrm{g} / 1$ (Table 2) shows that the serum levels of 10-formyltetrahydrofolate are significantly increased in many 
diseases. The reason for this is not immediately apparent but clearly has some significance in the interpretation of total serum folate levels. It is also apparent that there is some clinical variation, though the changes are small, in these levels throughout the day.

There seems to be a homeostatic mechanism for keeping the serum levels of 10-formyltetrahydrofolate constant in health (Ratanasthien et al., 1974), though clearly the levels must fall when the total serum folate diminishes to low levels. Since 10formyltetrahydrofolate is the coenzyme necessary for purine biosynthesis (Blakely, 1969) a substantial fall in its level could be one cause of megaloblastic anaemia. As was noted earlier, values of 10-formyltetrahydrofolate in such patients were significantly less than normal, confirming the observations made by DaCosta and Rothenberg (1971). Low values of serum 10-formyltetrahydrofolate could also result from a lack of vitamin $B_{12}$, for this coenzyme controls the rate of formation of 10-formyltetrahydrofolate from 5-methyltetrahydrofolate, and so bring about megaloblastic anaemia. The existence of a significant fall in 10-formyltetrahydrofolate can be derived from the data of DaCosta and Rothenberg (1971) in the serum of $B_{12}$-deficient subjects with megaloblastic anaemia.

There is now substantial evidence that there is considerably increased production of 10-formyltetrahydrofolate in rapidly proliferating cells. The enzymes of the folate cycle show marked variation with the rate of cell multiplication (Rosenblatt and Erbe, 1973), while studies of urinary folates in rats show that when large implanted Walker 256 tumours are present the relative amount of 10-formyltetrahydrofolate is increased while 5-methyltetrahydrofolate is decreased (Barford and Blair, 1975). This could be the reason for the increased levels found in psoriasis and leukaemia but the cause is not so clear in coeliac disease and rheumatoid arthritis.

A large proportion of the serum folate increase after oral administration of 5-methyl-5,6-dihydrofolate to pernicious anaemia subjects (Table 15) and the combined oral administration of it plus bicarbonate and of 5-methyltetrahydrofolate plus bicarbonate to normal subjects (Tables 6 and 9) is 5-methyl-5,6-dihydrofolate. This seems to persist without being metabolised and does not appear therefore to enter the folate metabolism pool in either normal subjects or in patients with pernicious anaemia. In the fasting state only patients with pernicious anaemia have a substantial portion of serum folate as this compound (Table 4). In all other cases the small proportion found could have been an analytical artefact.

Lastly, oral administration of 7,8-dihydrofolate
(Table 13) showed the same pattern of rapid conversion to serum 5-methyltetrahydrofolate with little change in serum 10-formyltetrahydrofolate levels as had been previously noted with tetrahydrofolate (Ratanasthien et al., 1974).

The major dietary folate compounds are 5-methyltetrahydrofolate and its derivatives. The data in this study suggest that their availability will depend on the amount of gastric acidity and of ascorbate in the intestinal chyme. In particular 5-methyl-5,6-dihydrofolate may not be available in the absence of ascorbate and in an acid stomach. Even when it does appear in the serum it seems unlikely that it will enter the folate metabolic pool since the serum levels tend to remain relatively constant for long periods.

These findings are particularly relevant, for total food folate is conventionally measured by $L$. case $i$ assay. Since our own observations suggest that about $50 \%$ of total food folate is 5-methyl-5,6-dihydrofolate (unpublished) measurements of food folate by $L$. casei assay may greatly exaggerate the utilisation of folate in food by man. If 5-methyl-5,6-dihydrofolate constitutes a major portion of food folate it may explain the increased proportion of this compound in the serum of patients with pernicious anaemia.

J. A. Blair thanks the Medical Research Council for a grant in support of this work.

\section{References}

Barford, P. A. and Blair, J. A. (1975). Novel urinary metabolites of folic acid in the rat. Proceedings of the Vth International Symposium on Chemistry and Biology, Pteridines, pp. 373-405. Walter de Gruyter, Berlin and New York.

Benn, A. N., Swan, C. H. J., Cooke, W. T., Blair, J. A., Matty, A. J., and Smith, M. E. (1971). Effect of intraluminal $\mathrm{pH}$ on the absorption of pteroylmonoglutamic acid. Brit. med. J., 1, 148-150.

Blair, J. A. and Pearson, A. J. (1974a). Kinetics and mechanism of the autoxidation of the 2-amino-4hydroxy-5,6,7,8-tetrahydropteridines. J. chem. Soc., (Perkin Trans. II), 1, 80-88.

Blair, J. A. and Pearson, A. J. (1974b). Some observations on the effect of light and solvent polarity on the kinetics of tetrahydrobiopterin autoxidation. J. chem. Soc. (Perkin Trans. II), 2, 1786-1787.

Blair, J. A., Pearson, A. J., and Robb, A. J. (1975). Autoxidation of 5-methyl-5,6,7,8-tetrahydrofolic acid. J. chem. Soc. (Perkin Trans. II), 1, 18-21.

Blair, J. A. and Saunders, K. J. (1970). A convenient method for the preparation of dl-5-methyltetrahydrofolic acid. Analyt. Biochem., (dl-5-methyl-5,6,7,8tetrahydropteroyl-L-monoglutamic acid). 34, 376-381 .

Blakely, R. L. (1969). The Biochemistry of Folic Acid and Related Pteridines, pp. 441-445, North Holland Publishing Company, Amsterdam and London.

Brown, J. P., Davidson, G. E., and Scott, J. M. (1973). Thin layer chromatography of pteroylglutamates and 
related compounds. J. Chromatogr., 79, 195-207.

DaCosta, M. and Rothenberg, S. P. (1971). Identification of an immunoreactive folate in serum extracts by radioimmunoassay. Brit. J. Haemat., 21, 121-130.

Donaldson, K. O. and Kereszetsy, J. C. (1962). Naturally occurring forms of folic acid III. Characterisation and properties of 5-methyldihydrofolate, an oxidation product of 5-methyltetrahydrofolate. J. biol. Chem., 237, 3815-3819.

Dransfield, E. (1973). The fate of folic acid and related compounds in the rat. Ph.D. thesis, University of Aston in Birmingham.

Futterman, S. (1963). Preparation and properties of dihydrofolic acid. Meth. Enzymol., 6, 801-802.

Gapski, G. R., Whiteley, J. M., and Huennekens, F. M. (1971). Hydroxylated derivatives of 5-methyl-5,6,7,8tetrahydrofolate. Biochemistry, 10, 2930-2934.

Gupta, V. S. and Huennekens, F. M. (1967). Preparation and properties of crystalline 5-methyltetrahydrofolate and related compounds. Arch Biochem. Biophys., 120, 712-718.

Herbert, V. (1966). Aseptic addition method for Lactobaccillus casei assay of folate activity in human serum. J. clin. Path., 19, 12-16.

Larabee, A. R., Rosenthal, S., Cathou, R. E., and
Buchanan, J. M. (1961). A methylated derivative of tetrahydrofolate as an intermediate in methionine biosynthesis. J. Amer. chem. Soc., 83, 4094-4095.

Leeming, R. J., Portman-Graham, H., Swan, C. H. J., and Blair, J. A. (1970). The application of tetrazolium bioautography to the identification of folic acid derivatives. J. clin. Path., 23, 411-413.

Pearson, A. J. (1974). Kinetics and mechanism of the autoxidation of tetrahydropterins. Chem. and Ind., 233-239.

Ratanasthien, K., Blair, J. A., Leeming, R. J., Cooke,W.T. and Melikian V. (1974). Folates in human serum. J. clin. Path. 27, 875-879.

Robinson D. R. (1971). The non-enzymatic hydrolysis of $\mathrm{N}^{5}, \mathbf{N}^{10}$-methenyltetrahydrofolic acid and related reactions. Meth. Enzymol., 18B, 716-725.

Rosenblatt, D. S. and Erbe, R. W. (1973). Reciprocal changes in the levels of functionally related folate enzymes during the culture cycle in human fibroblasts. Biochem. Biophys. Res. Commun., 54, 1627-1633.

Weir, D. G., Brown, J. P., Freedman, D. S., and Scott, J. M. (1973). The absorption of the diastereoisomers of 5-methyltetrahydropteroylglutamate in man: a carriermediated process. Clin. Sci. molec. Med., 45, 625-631. 